\title{
Versorgungsstrategien bei distalen Radiusfrakturen im Kindes- und Jugendalter
}

\author{
Dirk W. Sommerfeldt
}

\section{Zusammenfassung}

Der distale Radius stellt im Kindes- und Jugendalter über alle Altersstufen hinweg eine Prädilektionsstelle für unterschiedliche Frakturen dar. Biomechanische Schwachpunkte sind der diametaphysäre Übergang und die Wachstumsfuge. Häufig besteht eine Mitverletzung der distalen Ulna im Sinne der distalen Unterarmfraktur. Die konservative Frakturbehandlung stellt eine wesentliche Säule der unfallchirurgischen Therapie dar und führt bei der Mehrzahl der Frakturen in diesem Bereich zu sehr guten Ergebnissen. Bei tolerablen Fehlstellungen hilft das starke Wachstumspotenzial der distalen Epiphysenfuge dem Behandler, Achsabweichungen in der Hauptbewegungsebene innerhalb weniger Monate zu korrigieren [8]. Bei den instabilen und daher häufiger operativ zu versorgenden kompletten Frakturen im Bereich der Metaphyse und im meta-/diaphysären Übergang stehen mehrere Osteosyntheseverfahren gleichwertig mit unterschiedlichen Vor- und Nachteilen nebeneinander. Wichtig ist, dass - mit welchem Verfahren auch immer - eine rotationsstabile Retention des distalen Fragments erreicht wird [9]. Bei den Verletzungen mit Beteiligung der Wachstumsfuge sollte zwischen den komplikationsarmen E1- und E2Verletzungen und der häufiger mit Wachstumsstörungen assoziierten Crush-Verletzung unterschieden werden. Wichtig ist in dieser Situation eine gute Aufklärung und Nachsorge mit Verlaufskontrollen über 2 Jahre nach Trauma.

\section{Treatment Strategies for Distal Radius Fractures during Childhood and Adolescence}

During childhood and adolescence the distal radius is the most frequent site of injury showing various types of fractures. Biomechanical weak points are the diametaphyseal transition and the growth plates. The distal ulna is often injured concomitantly resulting in a distal forearm fracture. In most cases, fractures can be managed conservatively with excellent results. If there is only minor displacement, the strong growth potential of the distal growth plate of the radius is able to correct axis deviations in the sagittal plane within a few months [8]. As for unstable complete fractures in the region of the metaphysis and in the meta-/diaphyseal transition zone several equally useful techniques of osteosynthesis with varying advantages and disadvantages are available. Irrespective of the chosen procedure, a rotationally stable distal fragment should be achieved [9]. In cases of injuries involving the growth plates a distinction must be made between E1 and E2 injuries with lower complication rates and crush injuries of the growth plate that often lead to growth disturbances and posttraumatic deformities. In these situations comprehensive counselling and close follow-up with at least a 2-year follow-up are mandatory.

\section{Einleitung}

Distale Radiusfrakturen sind sowohl im Kindes- als auch im Jugendalter häufige Frakturen. Zum einen liegt dies an der Tatsache, dass die Hände im Fallen zum Abfangen des Sturzes eingesetzt werden, zum anderen daran, dass sich bis zum Verschluss der distalen Wachstumsfuge des Radius sowohl innerhalb des Knorpel-Knochen-Übergangs der Fuge selbst als auch im Bereich zwischen metaphysärer Spongiosa und diaphysärem Kortex ein biomechanischer Schwachpunkt (locus minoris resistentiae) befindet.

OP-JOURNAL 2012; 28: 246-251

(c) Georg Thieme Verlag KG Stuttgart · New York DOI http://dx.doi.org/10.1055/s-0032-1328026
Grundsätzlich ist diese Frakturlokalisation, die ja mehrere, sich deutlich voneinander unterscheidende Frakturtypen (s.u.) aufweist, bei noch vorhandenem Längenwachstum der Fuge (mindestens noch 2, besser 5 Jahre) eine Domäne der konservativen Therapie.

Dies gilt insbesondere für die stabilen Wulst- und Kompressionsverletzungen, aber auch für die in diesem Bereich selteneren Grünholzbrüche. Bei letzteren ist dann allerdings des Öfteren die primäre Reposition in Narkose mit anschließender Ruhigstellung im Unter- oder Oberarm-Cast, eventuell auch mit nachfolgender Keilung des Casts am 5.-7.Tag, erforderlich.
Es gibt jedoch auch in diesem Bereich eine Vielzahl von Situationen, altersund/oder frakturmorphologisch bedingt, die eine operative Versorgung erforderlich machen. Diese sollen im Folgenden ebenfalls näher beleuchtet werden und Empfehlungen ausgesprochen werden, welche OP-Technik sich bei welcher Fraktur gut einsetzen lässt und welche Verfahren eher nicht zum Erfolg führen werden.

\section{Diagnostik und Klassifikation}

Begleitverletzungen können insbesondere im Bereich des Ellenbogens bei der distalen Radius- bzw. Unterarmfraktur im Kindes- und Jugendalter für den weiteren Behandlungsverlauf entscheidende Bedeutung haben. 

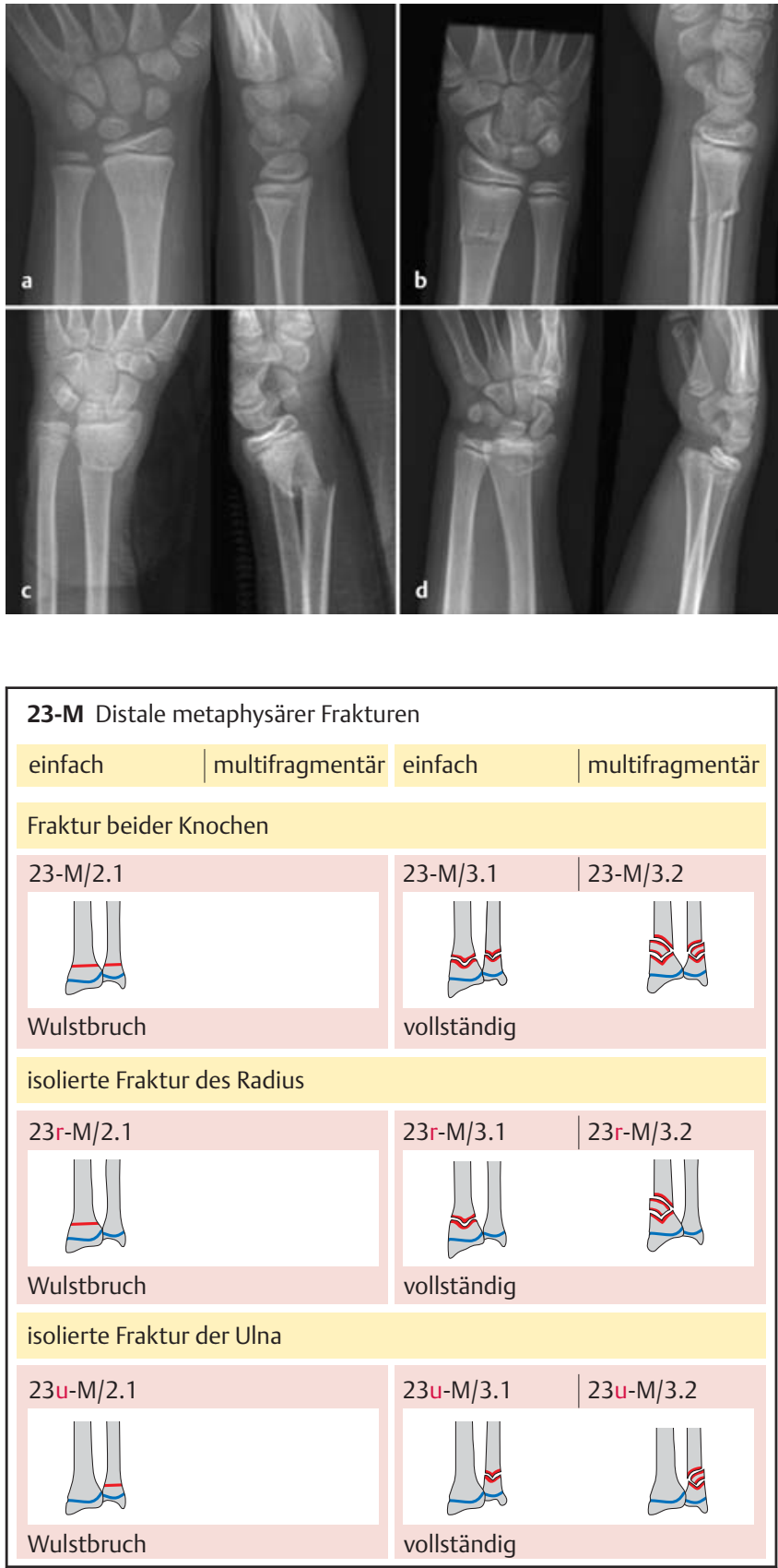

Es empfiehlt sich grundsätzlich nach genauer Anamnese und Untersuchung eine Standardröntgenaufnahme des Unterarms mit angrenzenden Gelenken in 2 Ebenen.

Je nach Befund können dann zusätzliche Zielaufnahmen des Ellenbogens und/ oder des Handgelenks durchgeführt werden. Selbstverständlich sollten alle erforderlichen Aufnahmen „kindgerecht", d. h. nach den Richtlinien der Kinderradiologie, angefertigt werden.

Es ist für den Behandler ein eindeutiger Vorteil, die Fraktur, die er therapieren
Abb. 1 a bis d

Die 4 Haupttypen der distalen Radiusfraktur im Kindes- und Jugendalter: a Wulstbruch, b Grünholzbruch, c komplette, bikortikale Fraktur, d Epiphyseolyse mit metaphysärem Keil. Sivertsen E. Classification of distal radius fractures in children: good inter- and intraobserver reliability, which improves with clinical experience. BMC Musculoskeletal Disorders 2012; 13 : published ahead of printing

Abb. 2 Frakturklassifikation der $\mathrm{AO}$ Pediatric Expert Group (PAEG) für den distalen Unterarm. Aus: Randsborg E,

ren Frakturen in stabile vs. instabile Frakturen (Wulstbruch vs. Grünholzbruch vs. komplette Fraktur beider Kortizes) [12] (Abb. 1). Für die Frakturen mit Beteiligung der Wachstumszone haben sich die Einteilungen nach Salter und Harris bzw. Aitken bewährt. Diese sind auch in die ausführlichere und umfassendere Klassifikation der Pediatric Expert Group (PAEG) der Arbeitsgemeinschaft für Osteosynthesefragen (AO) eingeflossen, die sich für wissenschaftliche Fragestellungen und Frakturregister eignet [13] (Abb. 2).

\section{Metaphysäre Extensions- und Flexionsbrüche}

Je nach Alter des Patienten und Grad der Krafteinwirkung kommen hier unterschiedliche Frakturformen vor, die sich bezüglich ihres sekundären Dislokationspotenzials zum Teil deutlich unterscheiden.

\section{Radiuswulstbruch (23 r-M/2.1 nach AO)}

Diese Kompressionsverletzung ist stabil und disloziert nahezu nie sekundär. Dementsprechend ist die Therapie in der Regel konservativ. Bis zur Vollendung des 3. Lebensjahrs, bei etwas voluminöseren Unterarmen auch bis zum Vorschulalter, wird der Oberarm-Cast aufgrund des besseren Halts empfohlen. Je nach Schwellung kann er als Schiene, als Sandwich-Cast oder zirkulär gespalten angelegt werden. Ab dem Schulalter ist der Unterarmcast ausreichend.

Da diese Frakturen stabil sind und bleiben, kann auf Röntgenkontrollen im Verlauf verzichtet werden.

Nach der Cast-Abnahme nach 2-3 Wochen je nach Alter wird die Funktion des Handgelenks klinisch untersucht und dokumentiert [2]. Sollten die Eltern oder Zuweiser auf eine Röntgenkontrolle drängen, kann der Ultraschall die Frage nach der Frakturkonsolidierung ebenso zuverlässig und ohne Strahlenbelastung beantworten [6].

\section{Grünholzfraktur}

Klassifikationen helfen für dieses Verständnis. Für den klinischen Alltag ist die Einteilung nach Dislokationsrichtung des distalen Fragments (also Extensionsoder Flexionsverletzung) und Grad der Dislokation in der Frontal- und Sagittalebene hilfreich, aber alleine nicht ausreichend. Sinnvoll, weil therapeutisch relevant, ist eine Einteilung der metaphysä-
Ist nur eine Kortikalis, meist auf der Zugseite der Fraktur, unterbrochen, spricht man von einer Grünholzfraktur. Auch eine komplette, bikortikale Fraktur, bei der das Periost noch nahezu zirkulär erhalten ist, ist biomechanisch als Grünholzbruch zu sehen. Sie sind im Schaftbereich deutlich häufiger als metaphy- 
sär. Die deutlich selteneren Flexionsfrakturen dislozieren häufiger sekundär als Extensionsfrakturen. Grünholzbrüche heilen zum Teil verzögert, bzw. dorsal und ventral unterschiedlich schnell. Sie haben dadurch ein erhöhtes sekundäres Dislokationspotenzial und haben daher im Gegensatz zum Wulstbruch auch radiologischen Kontrollbedarf im Verlauf (nach Reposition, 5., 10. und 20. Tag).

Je näher die Fraktur an der Wachstumsfuge liegt, umso höher ist das Korrekturpotenzial bezüglich einer Achsabweichung.

So können sich Fehlstellungen in der Sagittalebene von bis $\mathrm{zu} 30^{\circ}$ bis zum 10. Lebensjahr problemlos in einem akzeptablen Zeitraum korrigieren. Bei höhergradigen Achsabweichungen in der Sagittalebene, Fehlstellungen in der Frontalebene über $10^{\circ}$ und Seit-zu-SeitVersatz über einem Drittel der Schaftbreite sowie bei Patienten in der Adoleszenz sollte in Narkose reponiert werden [5]. Ein Überführen dieser Fraktur in eine rotationsinstabilere, komplette Fraktur mit dem sog. „Durchbrechen“ der noch stehenden Kortikalis ist nicht erforderlich. Bei guter Dreipunktabstützung (Abb. 3) im korrekt, also sparsam gepolsterten Cast kann auch diese Fraktur durch konservative Maßnahmen zur Ausheilung gebracht werden [1,10]. Auch die Technik der Gips- bzw. CastKeilung ist bei den sekundär oft dislozierenden Frakturen hilfreich und kann ab dem 5.-7. Tag nach Fraktur in der Regel ohne Narkose durchgeführt werden.

Die Refrakturrate ist im Vergleich zum Wulstbruch und zur kompletten Fraktur bei Grünholzbrüchen erhöht. Daher sind ausreichend lange Ruhigstellungszeiten (3-4 Wochen) und Sportkarenzen (4-8 Wochen je nach Sportart) wichtig.

Bewegungseinschränkungen des Handgelenks auch nach längerer Ruhigstellung lösen sich in diesem Alter nahezu immer von selbst, d.h. ohne die Notwendigkeit von physiotherapeutischen Maßnahmen, auf.

\section{Komplette, bikortikale Fraktur (23 r-M/3.1 nach AO)}

Diese Brüche treten im Kleinkindalter bei höherer Gewalteinwirkung und bei älteren Kindern und Jugendlichen vermehrt auf. Sie sind bei zerrissenem Periost naturgemäß instabiler als die o.a. Frakturformen und führen daher auch



Abb. 3 a bis d Grünholzbruch des Radius mit kompletter, bikortikaler Fraktur der Ulna bei intaktem Periost. a, b Unfallaufnahmen. c, d Nach Reposition und Oberarmcastanlage in leichter Flexionsstellung im Handgelenk und dadurch guter Dreipunktabstützung.

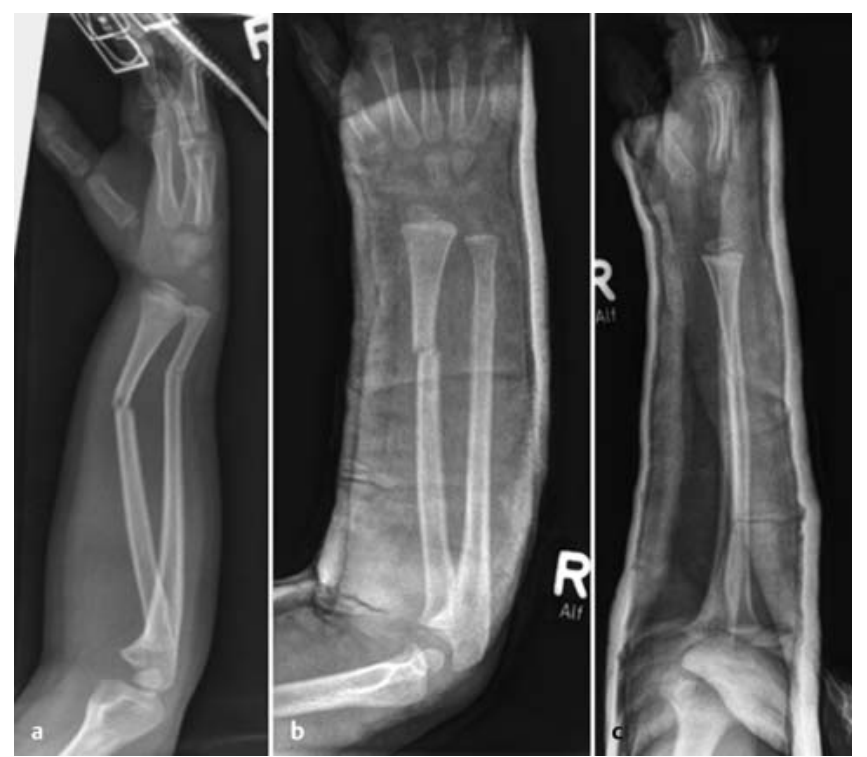

Abb. 4 a bis c Komplette, bikortikale, distale, diaphysäre Radiusfraktur mit Grünholzbruch der Ulna (bikortikal, aber intaktes Periost). a Unfallaufnahme. b, c Nach Reposition und Oberarmweißgipsanlage.

häufiger zu einer OP-Indikation. Offene Frakturen sowie Gefäß- und Nervenbeteiligung, vor allem des N. medianus, sind nicht selten und stellen eine Notfallindikation dar. Isolierte, vollständig dislozierte Radiusfrakturen sind selten, meist ist die Ulna mitfrakturiert.

Nach Reposition in Narkose nach den klassischen Repositionsprinzipien nach Böhler [1] bzw. Charnley [3] kann bei radiologisch im Bildwandler kontrollierter, stabiler Situation, also z.B. beim Querbruch, auch wieder konservativ behandelt werden (Abb. 4).

Ist die Fraktur nach Reposition instabil, sollte in gleicher Sitzung eines der folgenden Osteosyntheseverfahren zum Einsatz kommen.

\section{a) Bikortikale K-Draht-Osteosynthese}

Sie eignet sich naturgemäß eher für die distaleren, fugennahen Frakturen. Hierbei muss darauf geachtet werden, nach Eingehen über den Processus styloideus die Wachstumsfuge möglichst nur einmal zu perforieren, um das Risiko einer iatrogenen Wachstumsstörung gering zu halten. Auch ein Startpunkt metaphysär ist manchmal möglich und fugenschonend, wenn der Ranvier'sche Schnürring der Wachstumsfuge nicht tangiert wird. Ist das distale Fragment in der Frontalebene stabil und redisloziert nur in der Sagittalebene, reicht ein KDraht aus. Bei instabileren Frakturen oder einer größeren Trümmer- bzw. Stauchungszone müssen dann mindestens 2 Drähte eingebracht werden, um Rotationsstabilität zu erreichen (Abb. 5). 

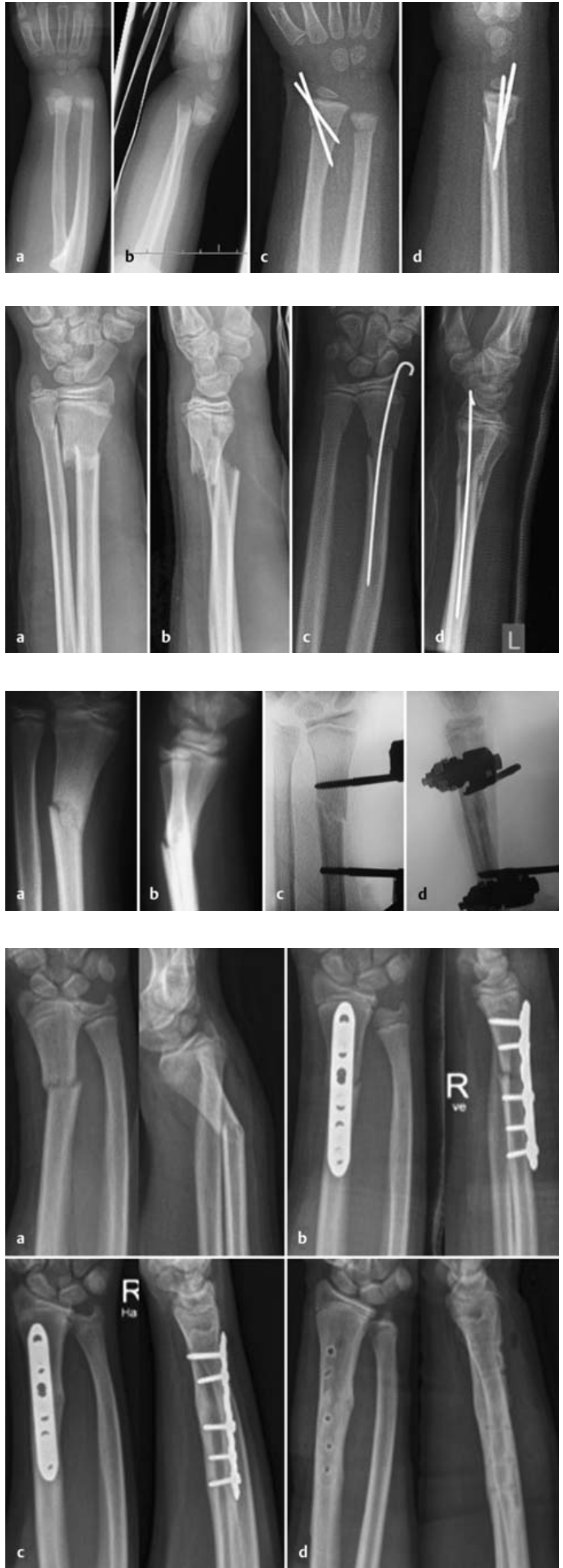

Abb. 5 a bis $d$

Komplette, bikortikale, distale, metaphysäre, grob dislozierte Unterarmfraktur. a, b Unfallaufnahmen. c, d Versorgung mit metaphysär eingebrachten KDrähten.

Abb. 6 a bis d Komplette, bikortikale, distale, metaphysäre, grob dislozierte Unterarmfraktur. a, b Unfallaufnahmen. c, $\mathbf{d}$ Versorgung mit metaphysär eingebrachtem KDraht.

Abb. 7 a bis d Komplette, bikortikale, distale Radiusfraktur mit intaktem radialem Periost. a, b Unfallaufnahmen. c, d Intraoperative Durchleuchtung bei Versorgung mit Fixateur externe.

Abb. 8 a bis d Komplette, bikortikale, isolierte, distale Radiusflexionsfraktur. a Unfallaufnahmen. b Postoperativ nach Plattenosteosynthese, 3,5er-LCP. c Konsolidiert, vor Metallentfernung (ME). d Nach ME. b) Intrafokale K-Draht-Osteosynthese n. Kapandji

Je weiter proximal die Fraktur liegt, umso schwerer wird die klassische Platzierung der K-Drähte. In der Technik nach Kapandji wird das distale Fragment nicht direkt angebohrt, sondern durch eine fächerförmige Anordnung der K-Drähte um das Fragment herum mit kortikaler Verankerung im proximalen Hauptfragment indirekt gehalten [7]. Auf den Verlauf der Strecksehnen der Langfinger und des Daumens muss hierbei besonders geachtet werden.

c) Transepiphysäre, intramedulläre K-Draht-Osteosynthese nach Py bzw. Desmanet

In der Technik nach Py wird epiphysär über den Processus styloideus und das Tuberculum Listeri eingegangen und der Draht dann im proximalen Hauptfragment möglichst weit nach intramedullär vorgeschoben [11]. Die Desmanet-Technik modifiziert dieses Vorgehen dadurch, dass statt des Tuberculums die dorsoulnare Kante des Radius als Eintrittspunkt für den 2. K-Draht gewählt wird [4]. Auch hier sind Strecksehnenverletzungen primär sowie sekundäre Rupturen als Komplikationen beschrieben. Beide Techniken sind ebenso wie die Kapandji-Technik ursprünglich zur Versorgung extraartikulärer distaler Radiusfrakturen des Erwachsenen entwickelt bzw. modifiziert worden. Sie haben sich aber gerade bei dieser Frakturlokalisation auch im Kindesalter bewährt [9].

Ebenso wie bei der Kapandji-Technik muss die Osteosynthese durch eine Unterarm-Cast-Schiene ergänzt werden (Abb. 6). Mit freundlicher Genehmigung Dr. Lieber, Tübingen.

d) Radialer, nicht gelenkübergreifender Fixateur externe

Vor allem bei offenen Frakturen, aber auch bei geschlossen nicht reponibler Fraktur kann der Fixateur externe eine gute Wahl sein. Je nach Lage der Fraktur können die Pins distal epi- oder metaphysär platziert werden, natürlich unter sicherer Schonung der Wachstumsfuge (Abb. 7). 


\section{e) Plattenosteosynthese}

Bei jugendlichen Patienten, bei Refrakturen, aber auch primär, z.B. als Rückzugsosteosynthese nach gescheitertem Versuch der K-Draht-Osteosynthese, ist eine in der Regel von streckseitig eingebrachte Kleinfragmentplatte in bestimmten Fällen eine sinnvolle Alternative. Nachteilig ist die Notwendigkeit der Metallentfernung in Narkose, Vorteil die sofortige Übungsstabilität (Abb. 8).

\section{f) Modifizierte ESIN-Osteosynthese nach Metaizeau}

Je weiter nach proximal die Frakturzone gelegen ist, umso verführerischer wird dieses Osteosyntheseverfahren, das ja die Therapie der Wahl für die Schaftfrakturen im Kindesalter darstellt. Durch entsprechendes Vorbiegen des elastischen Marknagels bzw. einen modifizierten Eintrittspunkt von dorsal über das Tuberculum Listeri kann auch bei metaphysären Frakturen die zwingend notwendige Dreipunktabstützung mit dem mittleren Abstützpunkt im distalen Fragment erreicht werden (Abb. 9) [9]. Gelingt dies nicht, so bleibt die Osteosynthese rotationsinstabil, es muss zusätzlich ruhiggestellt werden und der Vorteil der sofortigen Übungsstabilität dieses Verfahrens geht verloren. In solchen Fällen ist dann der Fixateur externe oder die Plattenosteosynthese, vor allem beim jugendlichen Patienten, eher indiziert.

\section{Frakturen mit Beteiligung der Wachstumsfuge}

Reine Epiphyseolyse (23 r-E/1 nach AO)

Diese Verletzung ist eher selten, meist ist ein metaphysärer Keil vorhanden. Je kleiner der Patient ist, umso kleiner ist auch der Knochenkern der distalen Radiusepiphyse, was die Diagnose in diesem Alter erschweren kann. In der Regel sind die Patienten jedoch über 10 Jahre alt und die Verletzung ist bereits klinisch sichtbar.

In der Pubertät lockert sich die Wachstumsfuge insgesamt auf und stellt dann einen biomechanischen Schwachpunkt dar.

In diesem Alter werden dann Frakturen mit Wachstumsfugenbeteiligung häufiger, metaphysäre Frakturen seltener.
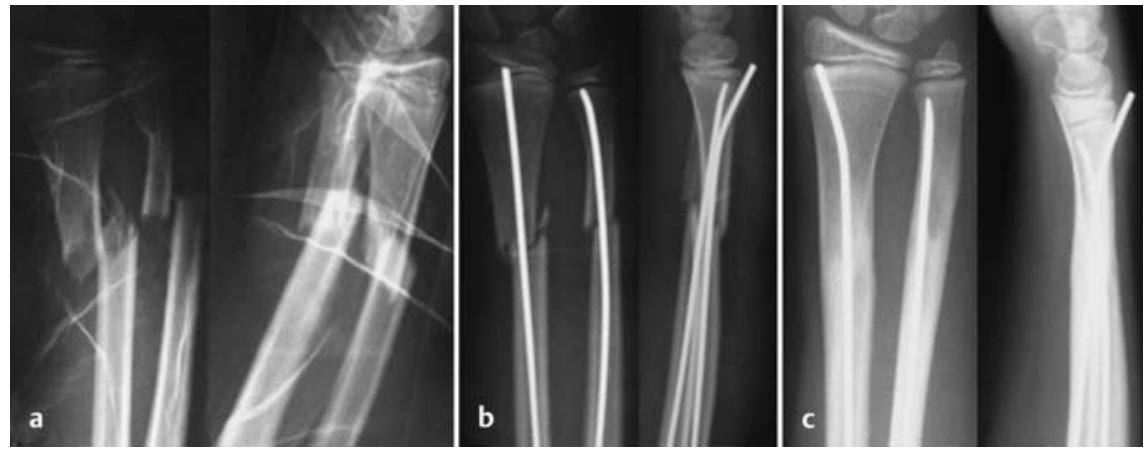

Abb.9a bis c Komplette, bikortikale, dislozierte, distale Unterarmfraktur. a Unfallaufnahmen. b Postoperativ nach ESIN. c Konsolidiert, vor Metallentfernung (ME).

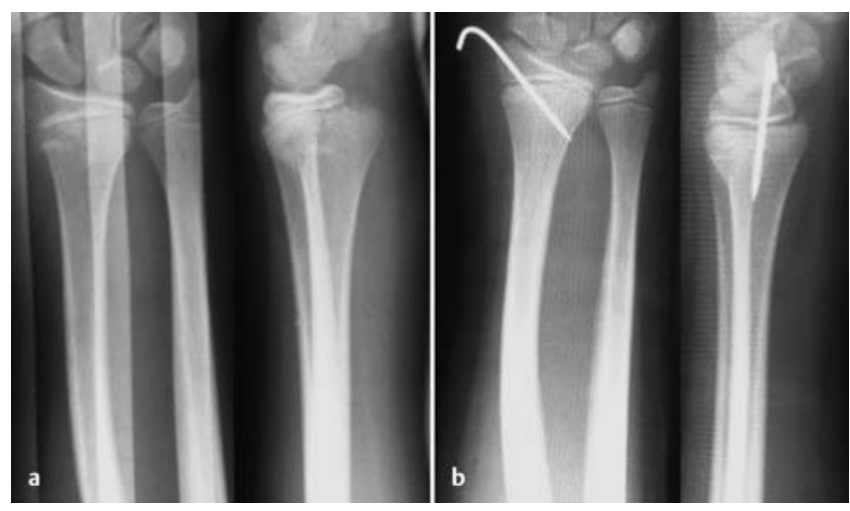

Abb. $10 \mathrm{a}$ und $\mathrm{b}$ Distale Radiusepiphyseolyse mit dorsalem, metaphysärem Keil. a Unfallaufnahmen. b Postoperativ nach schonender Reposition und transepiphysärer K-Draht-Osteosynthese.

Der N. medianus kann bei dieser Verletzung kompromittiert sein, die schonende Reposition im Aushang oder durch leichten Zug ist die Therapie der Wahl. Eine Osteosynthese ist selten erforderlich, wenn doch, reicht ein K-Draht, eingebracht über den Processus styloideus, immer aus. Die Inzidenz von Wachstumsstörungen ist gering, meist ist die für das Wachstum verantwortliche Zellschicht des Fugenknorpels unverletzt.

Wiederholte Manipulationen und forcierte Repositionsmanöver können zu einer iatrogenen Schädigung der Wachstumszone führen. Verlaufskontrollen bis zu 2 Jahre nach Trauma sowie eine Sicherungsaufklärung vom Behandler über die Möglichkeit eines solchen Folgeschadens müssen erfolgen.

\section{Epiphyseolyse mit metaphysärem Keil} (23 r-E/2.1 und 2.2 nach AO)

Diese Verletzung stellt den Löwenanteil der Frakturen im Bereich der Wachstumsfuge dar. Der metaphysäre, bei der Extensionsverletzung dorsale Keil kann unterschiedlich groß sein. Je weicher die Metaphyse in diesem Bereich ist, umso instabiler ist diese Fraktur nach Reposition und umso wahrscheinlicher wird eine K-Draht-Osteosynthese. Auch hier ist in der Regel $1 \mathrm{~K}$-Draht ausreichend, da die Fraktur meist nur in der Sagittalebene redisloziert (Abb. 10). Ausnahmen bestätigen auch hier die Regel.

Wachstumsstörungen treten hier aus den o.g. Gründen selten auf. Das empfindliche Stratum germinativum der Wachstumsfuge befindet sich epiphysär, die Fraktur verläuft metaphysär.

\section{Isoliert epiphysäre Fraktur (23 r-E/3)}

Diese Frakturform stellt eine absolute Rarität dar. Es kann der Processus styloideus radii betroffen sein oder im Sinne eines knöchernen Bandausrisses des distalen Radioulnargelenks die ulnare Kante des Radius. Meist ist die Wachstumsfuge beim Jugendlichen bereits verschlossen. Diese intraartikulären Verletzungen müssen stufenlos reponiert und meist durch kanülierte Schrauben retiniert werden. Ein partieller, vorzeitiger Verschluss der Wachstumsfuge ist theoretisch möglich und kann bei entsprechendem noch vorhandenem Wachstumspotenzial zu einem asymmetrischen Wachstum oder zu einem Wachstumsarrest mit ulnarem Vorschub führen. Daher sind auch hier Verlaufskontrollen über 2 Jahre zu empfehlen. 


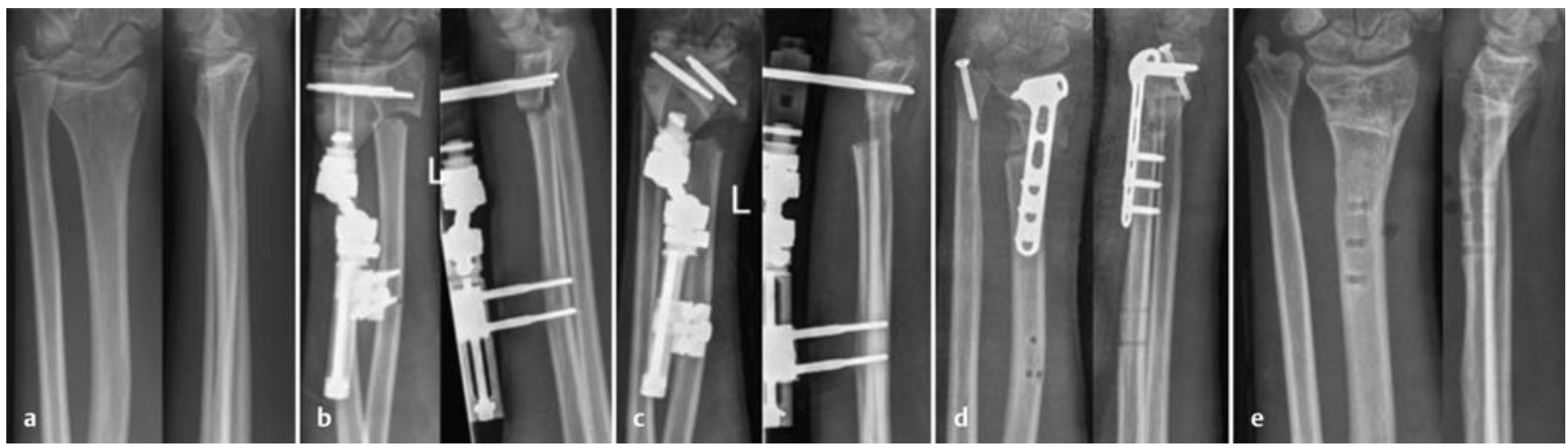

Abb. 11 a bis e Dreidimensionale Korrektur nach Crush-Verletzung der distalen Radiusepiphysenfuge im Kleinkindalter. a Erstvorstellung 10 Jahre nach Trauma mit aufgehobener Palmarflexion im Handgelenk. b Anlage unilateraler Fixateur externe mit Distraktionseinheit. c Radiusverlängerung um 4 cm, dann Durchblutungs- und Sensibilitätsprobleme. d Überbrückende Plattenosteosynthese zur Kallusreifung bei höherem Komfort und Ulnaepiphyseodese. e Derzeitiger Stand vor Ulnaverkürzungsosteotomie und freier Funktion.

\section{Epi-/metaphysäre Fraktur} (23 r-E/4.1 und 4.2 nach $A O)$

Ebenfalls eine äußerst seltene Verletzung, die nahezu immer im Adoleszentenalter bei bereits verschlossener Fuge auftritt. Auch hier gelten die Prinzipien für eine intraartikuläre Verletzung und die bereits genannten Wachstumskomplikationen, falls noch Restwachstum in der Fuge stattfinden sollte.

\section{Crush-Verletzung der Epiphysenfuge (23r-E/5)}

Diese Verletzungen sind initial schwer zu diagnostizieren, da im ersten Röntgenbild meist nichts zu sehen ist. Allenfalls können metaphysäre Stauchungszeichen mit Verdichtung der Trabekelstruktur erkennbar sein. Bei entsprechender Anamnese (Hochrasanztrauma) und Klinik (Schmerzen, Schwellung) kann diese Verletzung früh nur mittels MRT - und dann auch noch nicht sicher - ausgeschlossen werden.

Erst im Verlauf zeigen sich dann die Folgen dieser Verletzung mit Wachstumsarrest und posttraumatischer Deformität mit Radiusverkürzung. Da diese Patienten häufig jung sind und noch lange wachsen, werden nach dieser Verletzung oft mehrfache Verlängerungsoperatio- nen am distalen Radius notwendig (Abb. 11).

Auch an dieser Stelle sei daher nochmals auf die Notwendigkeit eines ausführlichen und gut dokumentierten Aufklärungsgesprächs über die Art der Verletzung, den weiteren Verlauf, notwendige Nachkontrollen und Möglichkeiten der Behandlung im Fall einer posttraumatischen Wachstumsstörung hingewiesen.

\section{Literatur}

${ }^{1}$ Böhler L. Die Techniken der Knochenbruchbehandlung. Wien: Maudrich; 1951

2 Bae D. Pediatric distal radius and forearm fractures. J Hand Surg [Am] 2008; 33: 19111923

${ }^{3}$ Charnley J. The closed Treatment of common Fractures. Edinburgh: Churchill Livingstone; 1950

${ }^{4}$ Desmanet $E$. L'ostéosynthèse par double embrochage souple du radius: traitement fonctrionnel du fractures de l'extrémité inférieure du radius: à propos d'un série de 130 cas. Ann Chir Main 1989; 8: 193-206

${ }^{5}$ Dietz H-G, Illing P, Schmittenbecher $P$ et al. Praxis der Kinder- und Jugendtraumatologie. Heidelberg: Springer; 2011

${ }^{6}$ Eckert K, Ackermann O, Schweiger B et al. Die Sonografie als sichere Alternative zur konventionellen Röntgendiagnostik bei distalen Unterarmfrakturen im Kindesalter. Z Orthop Unfall 2012; 150: 409-414

7 Kapandji $A$. L'osteosynthèse par double embrochage intra-focal: traitement fonctionnel des fractures non-articulaires de l'extremité infèrieure du radius. Ann Chir 1976; 30 : 903-908
${ }^{8}$ Kraus $R$. Distale Radiusfraktur im Wachstumsalter. Trauma Berufskrankh 2012; published ahead of printing

${ }^{9}$ Lieber J, Sommerfeldt D. Die diametaphysäre Unterarmfraktur im Kindesalter: Pitfalls und Empfehlungen in der Behandlung (Bd.4). Heidelberg: Springer; 2011

10 McQuinn A, Jaarsma R. Risk factors for redisplacement of pediatric distal forearm and distal radius fractures. J Pediatr Orthop 2012; 32: 687-692

${ }^{11}$ Py C. Zitiert in: Fractures of the distal radius. Eds. Safter P, Conney WP. Martin Dunitz Ltd. 1995: 59-61

12 Randsborg E, Sivertsen E. Classification of distal radius fractures in children: good interand intraobserver reliability, which improves with clinical experience. BMC Musculoskeletal Disorders 2012; 13: published ahead of printing

13 Slongo T, Audigé L, AO Pediatric Classification Group. Fracture and dislocation classification compendium for children: the AO pediatric comprehensive classification of long bone fractures (PCCF). J Orthop Trauma 2007; 10 (Suppl.): S135-S160

\section{Priv.-Doz. Dr. med.}

Dirk W. Sommerfeldt

Leiter der Abteilung für Kinder- und

Jugendtraumatologie

Altonaer Kinderkrankenhaus gGmbH

Bleickenallee 38

22763 Hamburg

dirk.sommerfeldt@

kinderkrankenhaus.net 